\title{
An Approximation Model for Sojourn Time Distributions in Acyclic Multi-Server Queueing Networks
}

\author{
Kevin R. Gue and Hyun Ho Kim \\ Department of Industrial \&3 Systems Engineering \\ Auburn University \\ Auburn, Alabama 36849
}

September 5, 2014

\begin{abstract}
We develop an approximation model for the sojourn time distribution of customers or jobs arriving to an acyclic multi-server queueing network. The model accepts general interarrival times and general service times, and is based on the characteristics of phase-type distributions. The model produces excellent results for multi-server networks with a small to medium number of workstations, but is less accurate when the number of workstations is large.
\end{abstract}

\section{Introduction}

For many service and order fulfillment settings, mean performance measures such as average work in process and expected flow time are insufficient as a means of understanding system performance and its implications for customer service. For example, rather than knowing that average flow time, or sojourn time, in an order fulfillment center is 50 minutes, managers might like to know what fraction of orders experience time in the system greater than, say, 3 hours. Answering such detailed questions requires a distribution of sojourn time, rather than just its mean value.

Much of the literature on sojourn time modeling is focused on calculating the mean flow time (or simply the waiting time) in single-stage queues or in queueing networks (Whitt, 1983). Workstations in these systems may have one or many servers. The subject of our work is calculating the distribution of sojourn time, a more difficult task. Specifically, we show how to approximate the sojourn time distribution of customers arriving to a serial or an acyclic queueing network, when interarrival times and processing times can be general.

Existing research on sojourn time modeling can be divided into four categories, according to whether the models address single-stage systems or networks, and whether they address single or 
multiple servers per workstation. The simplest case is single-stage, single-server systems. Neuts (1981) describes a matrix-geometric method to calculate the sojourn time distribution for a GI/G/1 system using phase type distributions. Luh and Zheng (2005) implemented Neuts' method in Mathematica. Sengupta (1989) used a bivariate Markov process to model waiting time and queue length distributions in a GI/PH/1 queue. His method is a "continuous analog" of the matrixgeometric method in Neuts (1981). Sengupta also showed that if the interarrival and service time distributions in a single-stage, single-server queue are phase-type, then the waiting time distribution is also phase-type.

Asmussen and O'Cinneide (1998) verify the same result for a single-stage, multi-server queue, in a paper extending Sengupta's work to the GI/PH/c case. Their model admits heterogeneous servers. Asmussen and Møller (2001) show how to calculate the waiting time distribution in a GI/PH/c and MAP/PH/c queue, for both homogeneous and heterogeneous servers. Whitt (1999) also addresses single-stage, multi-server systems, but examines state dependent waiting time distributions. For example, he shows how to compute the waiting time distribution for the $k$-th customer in line in a $c$-server, single-stage system. Rueda (2003) develop an approximation for the waiting time distribution of single-stage queues with non-stationary interarrival and processing time distributions.

Sojourn time distributions for queueing networks of single-servers have been addressed by Shanthikumar and Sumita (1988), You et al. (2002), and Yoon (1994). Shanthikumar and Sumita (1988) approximate the sojourn time distribution of an $\mathrm{M} / \mathrm{G} / 1$ queueing system as one of three phase-type distributions (generalized Erlang, exponential, and hyperexponential), based on a "service index," which is defined as the squared coefficient of variation of the total service time of an arbitrary job. Neuts (1981) showed that the convolution of phase type distributions is also phase type. You et al. (2002) used this observation to show how to calculate the sojourn time distribution for queueing networks, with general interarrival and service times. In a paper published in Korean, Yoon (1994) developed a method very similar to that of You et al.

Mandelbaum et al. (2002) develop models for Markovian multi-server queueing networks in which customers can abandon and retry to enter. They assume Markovian interarrival and service times. Gue and Kim (2012) develop a state-dependent sojourn time distribution model for remaining time of a customer in a multi-server, multi-stage queueing system, where the state of 
the system is the number of customers in each stage ahead of the customer of interest. The model allows general service times, but does not involve interarrival times because customers arriving after a customer is in the system do not affect its sojourn time.

Missing from the literature are models of sojourn time distributions for queueing networks with multiple servers, when interarrival and service times can take general distributions. We fill that void here. We believe ours is the first approximation model of sojourn time distributions for queueing networks of multi-server workstations.

Our model is based on the work of Neuts (1981), Asmussen and Møller (2001), and You et al. (2002): We use the bivariate Markov process of Asmussen and Møller to extract the mean and variance of waiting times for each multi-server workstation. We then construct phase type distributions for waiting time and service time based on those means and variances. Then we use the method of You et al. to construct an infinitesimal generator and initial probability vector for the network. With these we can calculate a sojourn time distribution for the network of multi-server queues. For reasons we discuss below, this model produces good results only when the inverse of the squared coefficient of variation $\left(1 / C^{2}\right)$ is close to an integer. We correct this weakness with a simple but effective interpolation scheme. We demonstrate the validity of our model by comparing it with results from a simulation model.

\section{Phase-type distributions}

Consider a Markov process on states $\{1, \ldots, m+1\}$, having infinitesimal generator

$$
\left[\begin{array}{cc}
\mathbf{Q} & \mathbf{Q}^{\mathbf{0}} \\
\mathbf{0} & 0
\end{array}\right] \text {, }
$$

where $\mathbf{Q}$ is an $m \times m$ matrix satisfying $Q_{i i}<0$, for $1 \leq i \leq m$, and $Q_{i j} \geq 0$, for $i \neq j$ (For consistency, we follow the notation of Neuts, 1981). $\mathbf{Q}^{0}$ is a column vector of size $m$ such that,

$$
\mathrm{Qe}+\mathrm{Q}^{\mathbf{0}}=\mathbf{0}
$$

where $\mathbf{0}$ is a row vector of zeros and $\mathbf{e}$ is a column vector of ones. The initial probability vector of $Q$ is $\boldsymbol{\beta}=\left(\beta_{1}, \beta_{2}, \ldots, \beta_{m}\right)$ such that $\boldsymbol{\beta e}=1$.

We are interested in the time until absorption into state $m+1$, whose distribution Neuts (1981) defines as phase-type. 
Lemma 1 (Neuts, 1981) The probability distribution $F(\cdot)$ of the time until absorption in the state $m+1$, corresponding to the initial probability vector $\left(\boldsymbol{\beta}, \beta_{m+1}\right)$ is given by

$$
F(x)=1-\boldsymbol{\beta} e^{\mathbf{Q} x} \mathbf{e} .
$$

Because $F(\cdot)$ is completely specified by $\boldsymbol{\beta}$ and $\mathbf{Q}$, the pair $(\boldsymbol{\beta}, \mathbf{Q})$ is called a representation of $F(\cdot)$.

Neuts (1989) provides the density function on $(0, \infty)$,

$$
f(x)=\boldsymbol{\beta} e^{\mathbf{Q} x} \mathbf{Q}^{0}=\boldsymbol{\beta} e^{\mathbf{Q} x}(-\mathbf{Q}) \mathbf{e} .
$$

For each distribution of service or waiting times in a network, we seek a phase-type approximation for which we can generate a matrix-analytic model of the CDF. We use the fact that finite convolutions of phase-type distributions are also phase-type (Neuts, 1981) to generate solutions for a queueing network, as in You et al. (2002).

The phase-type distribution is used to fit a general distribution based on the throughput rate $\lambda$ and the squared coefficient of variation $C^{2}$ of a given positive random variable $X$. Sauer and Chandy (1975), You et al. (2002), and Tijms (1994) showed different fitting methods that can convert a general distribution to a corresponding phase-type distribution based on the $C^{2}$ of the distribution.

A general distribution can be approximated with the Erlang distribution, the exponential distribution, or the hyperexponential distribution, based on its first and second moments. We follow the rule of You et al. (2002):

When $C^{2}<1$, we convert a general distribution to an Erlang distribution, $\operatorname{Erlang}(m, \mu)$, of order $m$. The infinitesimal generator is

$$
Q=\left[\begin{array}{cccccc}
-\mu & \mu & 0 & \cdots & 0 & 0 \\
0 & -\mu & \mu & \cdots & 0 & 0 \\
\vdots & \vdots & \vdots & \ddots & \vdots & \vdots \\
0 & 0 & 0 & \cdots & 0 & -\mu
\end{array}\right], Q^{0}=\left[\begin{array}{c}
0 \\
0 \\
\vdots \\
\mu
\end{array}\right]
$$

where $m=\left\lceil\frac{1}{C^{2}}\right\rceil$ and $\mu=m \lambda$. The phase-type representation of a general distribution is represented by $\boldsymbol{\beta}=(1,0, \ldots, 0)$ and $Q$. 
When $C^{2}>1$, we use the hyperexponential distribution with balanced means, $H E_{2}$, of order 2 . We use the following normalization for this process,

$$
\frac{p}{\mu_{1}}=\frac{q}{\mu_{2}}
$$

The phase-type representation of a general distribution is given by $\boldsymbol{\beta}=(p, q)$ and

$$
Q=\left[\begin{array}{cc}
-\mu_{1} & 0 \\
0 & -\mu_{2}
\end{array}\right], Q^{0}=\left[\begin{array}{l}
\mu_{1} \\
\mu_{2}
\end{array}\right]
$$

where $p=\frac{1}{2}\left(1+\sqrt{\frac{C^{2}-1}{C^{2}+1}}\right), q=1-p, \mu_{1}=2 p \lambda$ and $\mu_{2}=2 q \lambda$.

When $C^{2}=1$, we have an exponential distribution (Tijms, 1994) represented by $\boldsymbol{\beta}=1$ and $Q$,

$$
Q=-\lambda, Q^{0}=\lambda
$$

\section{Intuitive model}

We now describe our model, which extends the work of Asmussen and Møller (2001) and You et al. (2002) to produce an approximation of the sojourn time distribution for a network of multi-server queues. For the waiting time distribution of a single-stage, multi-server system, we follow Asmussen and Møller's method, then we assume independence and convolve waiting times and service times in the system based on the method of You et al. The model requires only the following input, which should be available in most real systems: mean and variance of processing times for each workstation and mean and variance of interarrival times to the system.

The procedure is:

1. Compute the arrival rate and SCV of an arrival process at each workstation using the Queueing Network Analyzer (QNA) method of Whitt (1983).

2. Approximate an interarrival time and service time distribution of each workstation $i$ as a corresponding phase-type distribution $\left(\boldsymbol{\alpha}_{\boldsymbol{i}}, A_{i}\right)$ and $\left(\boldsymbol{\beta}_{\boldsymbol{i}}, S_{i}\right)$ based on $C_{a}^{2}$ and $C_{s}^{2}$.

3. Compute the mean and variance of waiting time of each workstation $i$ using the method of Asmussen and Møller. Approximate the waiting time distribution of each workstation $i$ as a corresponding phase-type distribution $\left(\gamma_{i}, W_{i}\right)$ based on $C_{w}^{2}$. 
4. Assume independence and convolve all waiting times and service times sequentially to generate a phase-type representation of the sojourn time distribution $(\boldsymbol{\zeta}, K)$ using the convolution property of the phase-type distribution.

5. Solve $F(t)=P(T \leq t)=1-\zeta e^{K t} \mathbf{e},(t \geq 0)$ to obtain the cumulative distribution function $(\mathrm{CDF})$ of the sojourn time distribution.

\subsection{The flow rate and SCV of the arrival process}

We adopt the notation of Curry and Feldman (2009):

\begin{tabular}{cl}
\hline$n$ & the number of workstations in the general network \\
$\lambda_{i}$ & the average throughput rate of workstation $i$ \\
$\gamma_{i}$ & the mean arrival rate from external sources into workstation $i$ \\
$p_{j i}$ & the probability that a product flows from workstation $j$ to $i$ \\
$C_{d}^{2}(i)$ & the SCV of inter-departure times at workstation $i$ \\
$C_{a}^{2}(i)$ & the SCV of interarrival times at workstation $i$ \\
$C_{a}^{2}(0, i)$ & the SCV of external interarrival times at workstation $i$ \\
$C_{s}^{2}(i)$ & the SCV of service times at workstation $i$ \\
$\rho_{i}$ & the utilization of workstation $i$ \\
$c_{i}$ & the number of servers at workstation $i$ \\
\hline
\end{tabular}

To compute the waiting time distribution for each workstation, we must approximate the interarrival time and service time distributions as corresponding phase-type distributions. Thus, the throughtput rate $\lambda_{i}$ and $C_{a}^{2}(i)$ of interarrival times at each workstation $i$ must be calculated first, because the fitting distribution is based on the rate and $C^{2}$ of a general distribution.

The total throughput rate of workstation $i$ is

$$
\lambda_{i}=\gamma_{i}+\sum_{j=1}^{n} p_{j i} \lambda_{j} .
$$

From Curry and Feldman (2009, Property 5.4), the SCV of inter-departure times is approximately,

$$
C_{d}^{2}(i)=1+\left(1-\rho_{i}^{2}\right)\left(C_{a}^{2}(i)-1\right)+\rho_{i}^{2} \frac{\left(C_{s}^{2}(i)-1\right)}{\sqrt{c_{i}}}
$$


and the SCV of the interarrival times is approximately,

$$
C_{a}^{2}(i)=\frac{\gamma_{i}}{\lambda_{i}} C_{a}^{2}(0, i)+\sum_{j=1}^{n} \frac{\lambda_{j} p_{j i}}{\lambda_{i}}\left[p_{j i} C_{d}^{2}(j)+1-p_{j i}\right]
$$

From these expressions, we can approximate the interarrival time distribution and service time distribution of each workstation $i$ as a corresponding phase-type distribution $\left(\boldsymbol{\alpha}_{\boldsymbol{i}}, A_{i}\right)$ and $\left(\boldsymbol{\beta}_{\boldsymbol{i}}, S_{i}\right)$ based on the throughput rate and SCV.

\subsection{Waiting time for a single stage queue with multiple servers}

Asmussen and Møller (2001) proposed a method to compute the steady-state waiting time distribution in a multi-server queue, with each server having a phase-type service time distribution. They used matrix-analytic methods based on the method of Sengupta (1989) and Asmussen and O'Cinneide (1998). Asmussen and O'Cinneide (1998) showed that the GI/PH/c waiting time is always phase-type, and that the number of phases for the homogeneous case is

$$
\left(\begin{array}{c}
m+c-1 \\
c
\end{array}\right)
$$

where $m$ is the number of phases of the service time distribution.

They solve the system using a bivariate Markov process $\left\{X^{t}, N^{t} ; t \geq 0\right\}$ to model the all busy period, where $X^{t}$ is the time since arrival of the last customer to enter service in the all-busy-period, and $N^{t}$ is the current phase in which the server is working. They proved that the steady state density $\pi$ of the Markov process $\left\{X^{t}, N^{t}\right\}$ is matrix exponential:

$$
\pi(x)=\pi(0) \exp (R x), x>0
$$

where $R$ is the rate matrix. Their procedure is as follows:

The rate matrix $R$ satisfies the non-linear integral equation

$$
R=S+\int_{0}^{\infty} \exp (R u) A^{(j u m p)} H(d u)
$$

where the matrix $S$ contains transition rates without service completion, $A^{(j u m p)}$ contains transition rates with service completion in $\left\{N_{t}\right\}$, and $H(d u)$ is the distribution function of the interarrival times. 
The matrix $R$ is solved by iterative procedure and the phase-type representation of the waiting time of the single stage is defined by $(\boldsymbol{\rho}, G)$,

$$
\rho_{i}=\frac{\alpha_{i} \varphi_{i}}{\alpha \varphi}, G_{i j}=\frac{\alpha_{j} R_{j i}}{\alpha_{i}}
$$

where $\boldsymbol{\alpha}=-\pi(0) R^{-1}$ and $\boldsymbol{\varphi}=(R-S) \mathbf{e}$.

Finally, the mean and variance of the waiting time distribution are computed from the first and second moment, where the $n$-th moment is

$$
E\left(X^{n}\right)=(-1)^{n} n ! \rho G^{-n} \mathbf{e}
$$

\subsection{Approximation of the sojourn time distribution of a queueing network}

Now we are ready to apply the convolution property of phase-type distributions. Neuts (1981) showed that if $F(\cdot)$ and $G(\cdot)$ are both continuous phase-type distributions with representations $(\boldsymbol{\alpha}, A)$ and $(\boldsymbol{\beta}, S)$ of orders $m$ and $n$, then the convolution $F * G(\cdot)$ is a phase-type distribution with representation $(\boldsymbol{\gamma}, L)$, where the infinitesimal generator $L$ and initial probability vector $\gamma$ are

$$
\begin{gathered}
L=\left[\begin{array}{cc}
A & A^{0} \boldsymbol{\beta} \\
0 & S
\end{array}\right], \\
\gamma=\left[\boldsymbol{\alpha}, \alpha_{m+1} \boldsymbol{\beta}\right] .
\end{gathered}
$$

The sojourn time is a sum of random variables for waiting times and service times, which we assume are independent. We construct the initial probability vector and the infinitesimal generator of the sojourn time by convolving the distributions of waiting times $\left(\gamma_{i}, W_{i}\right)$ and service times $\left(\boldsymbol{\beta}_{\boldsymbol{i}}, S_{i}\right)$ by order in the network. For example, for a $k$ station serial line, the infinitesimal generator $K$ and initial probability vector $\zeta$ are

$$
K=\left[\begin{array}{cccccc}
W_{1} & W_{1}^{0} \beta_{1} & 0 & \cdots & 0 & 0 \\
0 & S_{1} & S_{1}^{0} \gamma_{2} & \cdots & 0 & 0 \\
\vdots & \vdots & \vdots & \ddots & \vdots & \vdots \\
0 & 0 & 0 & \cdots & W_{k} & W_{k}^{0} \beta_{k} \\
0 & 0 & 0 & \cdots & 0 & S_{k}
\end{array}\right],
$$


The cumulative distribution function $(\mathrm{CDF})$ and the probability density function $(\mathrm{PDF})$ are

$$
\begin{gathered}
F(t)=1-\zeta e^{K t} \mathbf{e},(t \geq 0) \\
f(t)=\zeta e^{K t} K^{0}=\zeta e^{K t}(-K) \mathbf{e},(t \geq 0)
\end{gathered}
$$

In a more general queueing network, flow between workstations depends on product routings. We estimate the sojourn time distribution for a random job by approximating the CDF of all possible "serial lines" (paths in the network) and mixing those CDFs according to the probabilities of taking those paths. Because our model is based on serial line analysis, we are able to model only acyclic queueing networks. We give an example in a later section.

\subsection{Numerical results}

To validate the intuitive model, we compare it with results of a simulation built in Arena 10.0. We assume processing and interarrival times in the simulation model are Gamma distributed.

Example 1 Consider a serial line of 3 workstations, with 6 servers per workstation (Figure 1). Information on the interarrival and processing times are given in Table 6 .

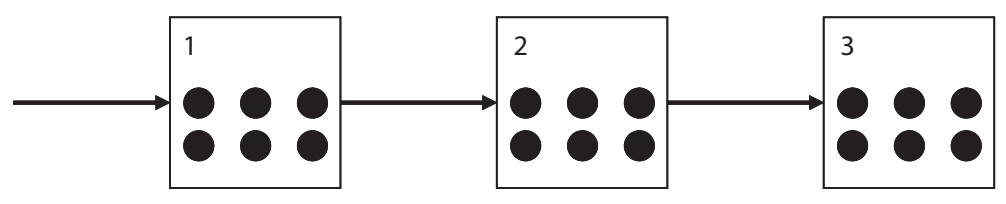

Figure 1: A serial line with 3 workstations. Workers are indicated by black dots.

We can approximate the interarrival time and three service time distributions as $\operatorname{Erlang}(m, \mu)$ distributions according to the rule of You et al. (2002) because all $C^{2}$ are less than 1 . Note that in this case all $\left\lceil\frac{1}{C^{2}}\right\rceil$ and $1 / C^{2}$ have the same value of 2 . The mean sojourn times in Table 6 show that the intuitive model is close to the simulation result. Figure 2 shows the sojourn time distributions from the intuitive model and from the simulation, and we see nice agreement between them.

Example 2 Consider the same 3-workstation serial line with $C_{a}^{2}=0.8, C_{s}^{2}(1)=0.6, C_{s}^{2}(2)=0.5$ and $C_{s}^{2}(3)=0.9$. 
Table 1: A comparison of the mean sojourn times between the intuitive model and simulation for a 3 -workstation serial line, when all $1 / C^{2}$ are integers.

\begin{tabular}{|c|c|c|c|c|c|c|}
\hline & \multirow{2}{*}{$E[T]$} & \multirow{2}{*}{$C^{2}$} & \multirow{2}{*}{$\rho$} & \multicolumn{2}{|c|}{ Mean sojourn time (hrs) } & \multirow{2}{*}{$\%$ difference } \\
\hline & & & & Simulation & Intuitive & \\
\hline Interarrival & 0.5 & 0.5 & & \multirow{4}{*}{5.79} & \multirow{4}{*}{5.77} & \multirow{4}{*}{-0.23} \\
\hline Workstation 1 & 1.8 & 0.5 & 0.6 & & & \\
\hline Workstation 2 & 2.2 & 0.5 & 0.73 & & & \\
\hline Workstation 3 & 1.5 & 0.5 & 0.5 & & & \\
\hline
\end{tabular}

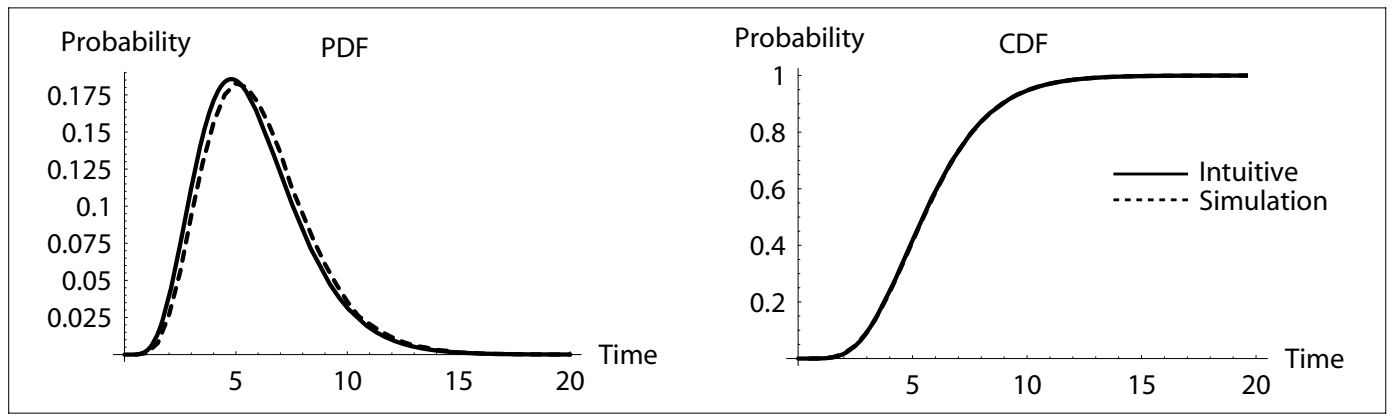

Figure 2: Sojourn time distributions for Example 1, when all $1 / C^{2}$ are integers. 
The mean sojourn time and sojourn time distribution of this system are shown in Table 2 and Figure 3 respectively. The percent difference of mean sojourn time between the two models is much higher, and the distributions appear not to be so similar. The intuitive model produces the same result when $1 / C^{2}$ falls into the interval $\left(\left\lfloor\frac{1}{C^{2}}\right\rfloor,\left\lceil\frac{1}{C^{2}}\right\rceil\right)$, but we get a new simulation result with a new $C^{2}$. For example, although $C_{a}^{2}$ changed from 0.5 to 0.8 , the interarrival time distribution is still fitted with the same $\operatorname{Erlang}(m, \mu)$ distribution, because $\left\lceil\frac{1}{C^{2}}\right\rceil$ is the same for both cases.

Table 2: A comparison of the mean sojourn times between the intuitive model and simulation when all $1 / C^{2}$ are not integers.

$$
E[T] \quad C^{2} \quad \rho \frac{\text { Mean sojourn time (hour) }}{\text { Simulation Intuitive }} \% \text { difference }
$$

\begin{tabular}{|c|c|c|c|c|c|c|}
\hline Interarrival & 0.5 & 0.8 & & \multirow{4}{*}{5.97} & \multirow{4}{*}{5.77} & \multirow{4}{*}{-3.35} \\
\hline Workstation 1 & 1.8 & 0.6 & 0.6 & & & \\
\hline Workstation 2 & 2.2 & 0.5 & 0.73 & & & \\
\hline Workstation 3 & 1.5 & 0.9 & 0.5 & & & \\
\hline
\end{tabular}

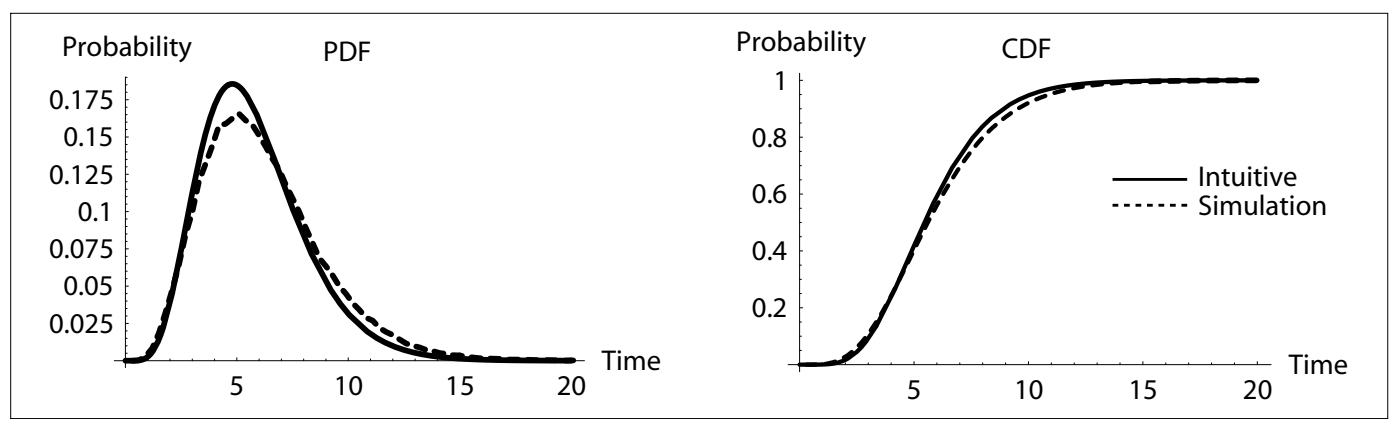

Figure 3: A comparison of sojourn time distributions between the intuitive model and simulation when all $1 / C^{2}$ are not integers.

This example illustrates a characteristic of the intuitive model that we have confirmed with more thorough testing: the intuitive model produces good results only when $1 / C^{2}$ is close to an integer value (under the $C^{2}<1$ condition). When $1 / C^{2}$ is between integer values (say, for example, when $C^{2}=0.75$ ), the intuitive model produces less satisfactory results. In fact, the model produces 
the same sojourn time distribution for all values of $C^{2}$ having a common value of $\left\lceil\frac{1}{C^{2}}\right\rceil$.

\section{Interpolation model}

A naïve attempt to correct for this deficiency by replacing the ceiling operator with a round( ) function produced even worse results. We introduce, then, the following interpolation scheme:

1. Produce CDF $F(t)$ using $\left\lceil\frac{1}{C^{2}}\right\rceil$ phases for each workstation.

2. Produce CDF $G(t)$ using $\left\lfloor\frac{1}{C^{2}}\right\rfloor$ phases for each workstation.

3. Compute the mixed CDF, $H(t)=\alpha G(t)+(1-\alpha) F(t)$, where $\alpha$ is an interpolation coefficient.

The interpolation coefficient $\alpha$ is based on two observations. First, if $1 / C^{2}$ for interarrival and processing times is close to $\left\lceil\frac{1}{C^{2}}\right\rceil$, then we should expect $\alpha$ to be close to zero; if $1 / C^{2} \approx\left\lfloor\frac{1}{C^{2}}\right\rfloor$, $\alpha$ should be close to one. Second, interarrival times and workstation processing times will have different levels of influence on the sojourn time distribution, depending on whether they appear early or late in the (serial) process. We would expect interarrival times and earlier processing times to have a greater effect on variability than later processing times, for example.

These observations lead us to a regression model of the squared coefficients of variation (independent variables) for each distribution in the process. To address the first observation, we define a fitting coefficient for each interarrival and processing time distribution. The fitting coefficient is an interpolation value reflecting the nearness of the squared coefficient of variation to successive values of $C^{2}$ when $1 / C^{2}$ takes on an integer value. For example, if the SCV of the arrival process $C_{a}^{2}=0.8$, we compute a fitting coefficient $\delta_{0}=0.6$, because $0.5+0.6(1-0.5)=0.8$, or said another way, 0.8 is $60 \%$ of the way between 0.5 and 1 . We can compute fitting coefficients $\delta_{k}$, which serve as the independent variables in the regression, for each workstation $k$ in a similar fashion.

Next, we determine the different levels of influence of the fitting coefficients with a regression model, where the dependent variable is the mean sojourn time produced by the intuitive model and the independent variables are the SCVs. For the data, we solve the intuitive model for all possible combinations of three SCVs $(1,1 / 2,1 / 3)$, each corresponding to an integer value of $1 / C^{2}$. For example, the regression for two workstation serial lines must consider three distributions - the 
interarrival times and service times for two workstations, and there are $3^{3}=27$ combinations. We excluded SCVs less than 1/3 because they have very little effect on mean sojourn time.

There is one last detail: we must consider the possible effects of utilization on the mean sojourn times and therefore on the coefficients $\omega_{i}$ produced by the regression. We ran two cases $(\rho=$ 0.5, 0.85 for each workstation in the system), and found that the resulting coefficients $\omega_{i}$ are different. Which values to use, given that we seek a single set of coefficients for any serial line of a specified length? We answered this question by using for each distribution the average of the two values, for two reasons: (1) For most systems in practice, the utilization of workstations in a system are different anyway, and (2) the results of the model are not very sensitive to the precise values of the $\omega_{i}$ 's.

Table 3 contains results of the regression analysis, which are the weight coefficients $\omega_{i}$ for 2 , 3 , and 4 station serial lines. In general, the interarrival times and the first workstation account for the largest influence on the sojourn time distribution, and the influence decreases as it goes downstream.

Table 3: The weight coefficient of serial lines with 2, 3 and 4 workstations.

\begin{tabular}{cccccc} 
Workstations & $\omega_{0}$ & $\omega_{1}$ & $\omega_{2}$ & $\omega_{3}$ & $\omega_{4}$ \\
\hline 2 & 0.493 & 0.313 & 0.194 & & \\
3 & 0.412 & 0.292 & 0.174 & 0.123 & \\
4 & 0.353 & 0.262 & 0.180 & 0.123 & 0.081 \\
\hline
\end{tabular}

Because the interpolation scheme incorporates the position of a particular distribution in the process, it also addresses, in a loose way, the issue of dependence between random variables. For example, results of the regression model assigned less weight to workstations further downstream (or more precisely, their distributions; see Table 3), presumably because variation from these workstations has less effect on the sojourn time distribution than does similar variation upstream. We also observe that this phenomenon would hold irrespective of the particular fitting distribution, and so any such choice would likely need some modification. 
Now that we have the fitting coefficients $\delta_{i}$ and weight coefficients $\omega_{i}$. We calculate the interpolation coefficient

$$
\alpha=\sum_{i=0}^{k} \omega_{i} \delta_{i},
$$

where index zero represents interarrival times. We should emphasize the conditions for which the $\omega_{i}$ 's in Table 3 are relevant: These coefficients should be appropriate for any serial lines of these lengths for which the Gamma distribution is a reasonable approximation of processing times.

Before fully testing the interpolation model, we need to show that it is better than the simpler, intuitive model. Figure 4 shows the differences in mean sojourn times among the simulation and the two approximation models when each workstation has the same utilization $\rho=0.5$ and SCVs. The interpolation and intuitive models are the same when all $1 / C^{2}$ are integers, but when they are not, the interpolation model is closer to the simulation. The intuitive model also corrects the deficiency noted in Table 2: for this problem, the difference in means is reduced to $-1.26 \%$ (mean sojourn time $=5.90$ hours).

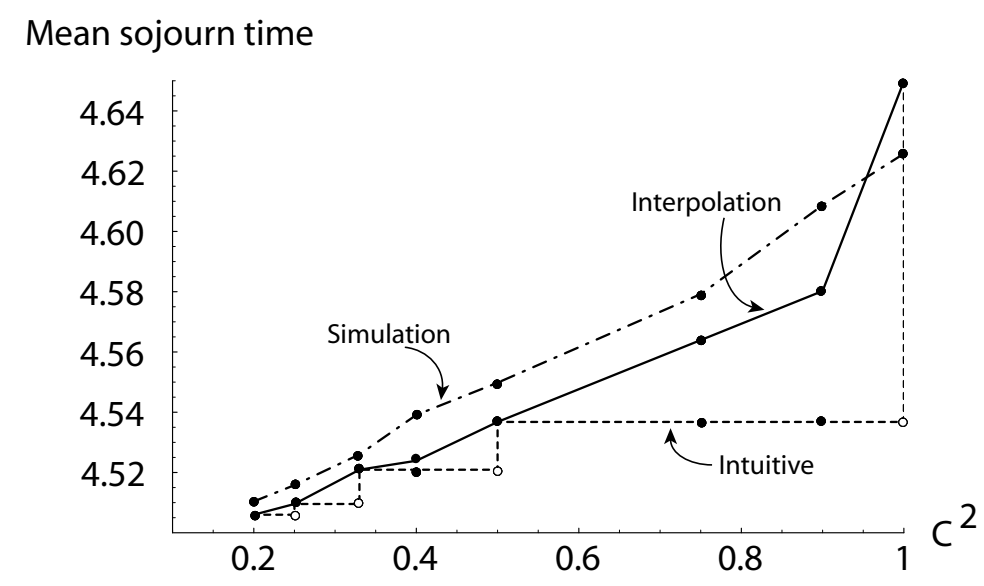

Figure 4: A comparison of mean sojourn times among three models $(\rho=0.5)$. Dots indicate data; lines are inserted for visual clarity only.

\subsection{Validation}

To test the performance of the model, we consider four queueing networks: The first is a small serial line with 3 workstations, as in previous examples; the second is a simple acyclic queueing 
network with 4 workstations (Figure 5), the third is a large-scale system with almost 100 servers in 3 workstations, and the last is a long serial line with 8 workstations. We wish to confirm the effectiveness of the interpolation model on a basic serial line and general queueing network through the first two experiments. With the second two, we investigate the computational time and accuracy of the interpolation model as the number of servers and workstations increases. In Figure 5, the black circles in each workstation represents servers, and a customer or order departing from the first workstation selects its follow on workstation with probability $p$.

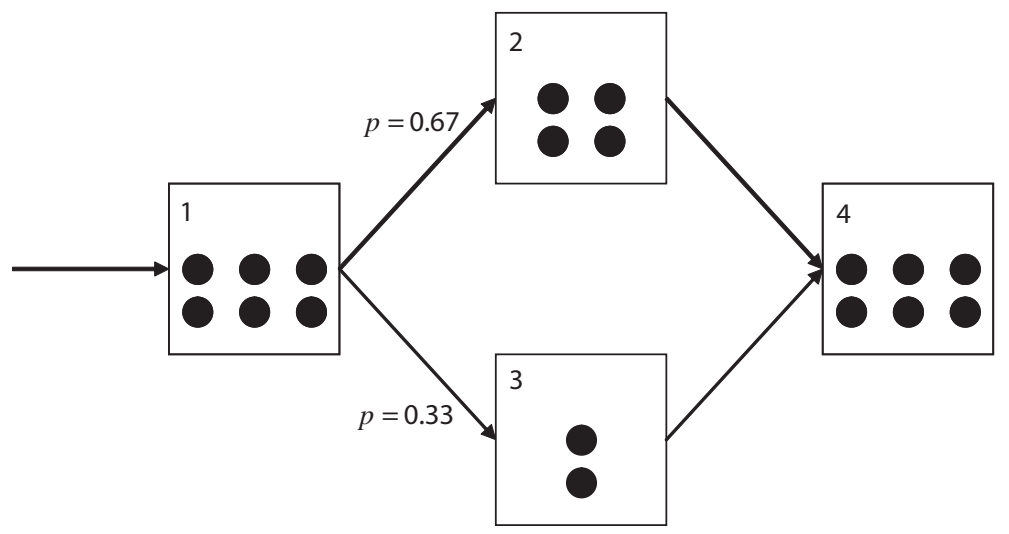

Figure 5: A general network with 4 workstations.

Example 3 Consider a 3-workstation serial line with 6 servers per workstation (Figure 1). All servers have the same capacity $(E[T]=1.5)$, and interarrival times are adjusted to produce the appropriate utilization $(\rho=0.5,0.85)$.

We compare simulation and model results over different values of SCV for interarrival and processing times. Table 4 shows the mean sojourn times for the interpolation model and simulation. The percent difference of the mean sojourn time between the two models under high utilization is a little bit higher than in the low utilization case. We conjecture that this is due to the amount of waiting time at each workstation. In the low utilization case, there is very little waiting in front of each workstation, so inaccuracies in the waiting time model do not have much effect on the estimate of the mean sojourn time. In the high utilization case, model inaccuracies have a greater effect. However, we see that the percent differences between the interpolation model and the simulations are still less than 2 percent. Notice that this statement only pertains to the mean, when what we 
seek is the distribution; that is, agreement in the means is only an indication that the model is effective, not a proof.

Table 4: Mean sojourn times for the interpolation and simulation models of the serial line.

\begin{tabular}{cccccccc}
\hline$\rho$ & $C_{a}^{2}$ & $C_{s}^{2}(1)$ & $C_{s}^{2}(2)$ & $C_{s}^{2}(3)$ & Interpolation & Simulation & \% difference \\
\hline \multirow{4}{*}{0.85} & 1 & 1 & 1 & 1 & 7.63 & 7.57 & 0.83 \\
\cline { 2 - 8 } & 0.75 & 0.75 & 0.75 & 0.75 & 6.77 & 6.77 & -0.04 \\
\cline { 2 - 8 } & 0.57 & 0.7 & 0.6 & 0.9 & 6.44 & 6.43 & 0.05 \\
\hline & 0.45 & 0.75 & 0.4 & 0.9 & 6.13 & 6.19 & -0.35 \\
\hline & 0.4 & 0.6 & 0.26 & 0.8 & 5.88 & 5.89 & -0.13 \\
\hline & 0.33 & 0.33 & 0.33 & 0.33 & 5.57 & 5.48 & 1.71 \\
\hline \multirow{3}{*}{0.5} & 1 & 1 & 1 & 1 & 4.65 & 4.62 & 0.51 \\
\hline & 0.75 & 0.75 & 0.75 & 0.75 & 4.53 & 4.58 & -0.30 \\
\hline & 0.45 & 0.7 & 0.6 & 0.9 & 4.57 & 4.55 & 0.29 \\
\hline & 0.4 & 0.6 & 0.26 & 0.8 & 4.53 & 4.53 & -0.03 \\
\hline & 0.33 & 0.33 & 0.33 & 0.33 & 4.52 & 4.53 & -0.23 \\
\hline
\end{tabular}

Figures 6-9 show the distributions for representative cases. We use the Anderson-Darling test (Law and Kelton, 2000) to check the graphical agreement of the distributions between simulation and approximation results. We also include the percent difference of the $90^{t h}$ and $95^{\text {th }}$ percentiles. As shown in Table 5, we accept that there is no difference between the distributions based on the A-D test, and the percent differences of the $90^{\text {th }}$ and $95^{\text {th }}$ percentiles are less than 3 percent.

Figures 6-9 indicate that the model consistently has a higher peak in the PDF than does the simulation model. We believe this is due to the approximation of waiting time distributions, which for all cases we tested had $C^{2}>1$, and therefore led us to the hyperexponential distribution as an approximation. Because the hyperexponential distribution is skewed to the left, it may cause our distributions of sojourn time also to be skewed to the left. 


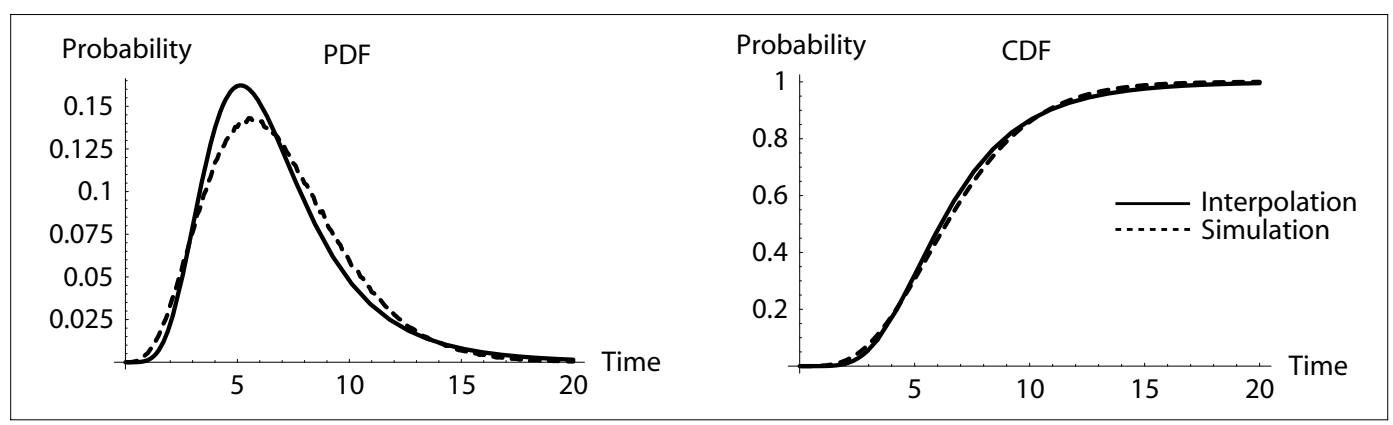

Figure 6: $\mathrm{CDF}$ and PDF of the serial line $\left(\rho=0.85, C^{2}=0.75,0.75,0.75,0.75\right)$.

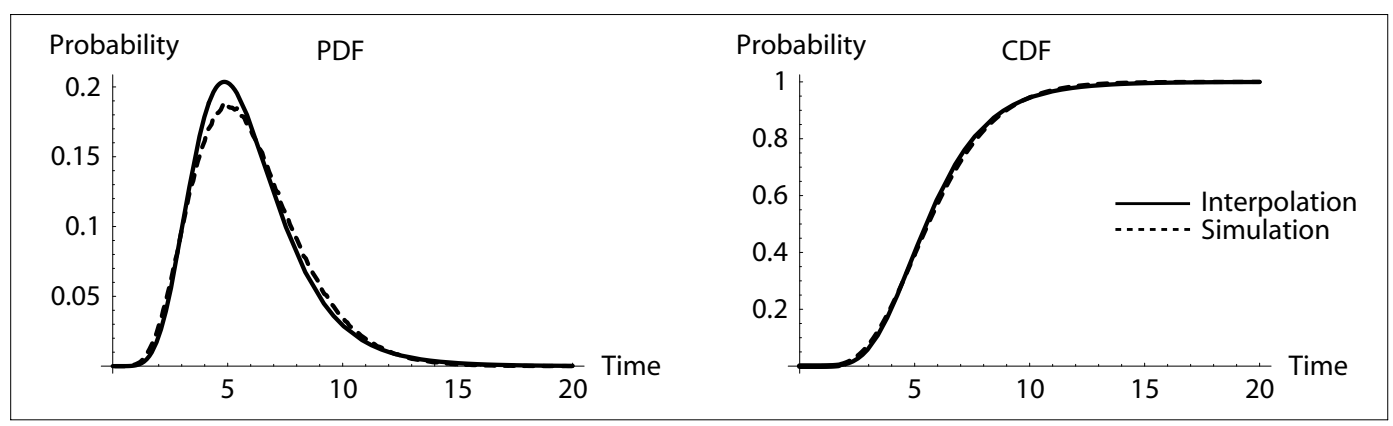

Figure 7: $\mathrm{CDF}$ and PDF of the serial line $\left(\rho=0.85, C^{2}=0.4,0.6,0.26,0.8\right)$.

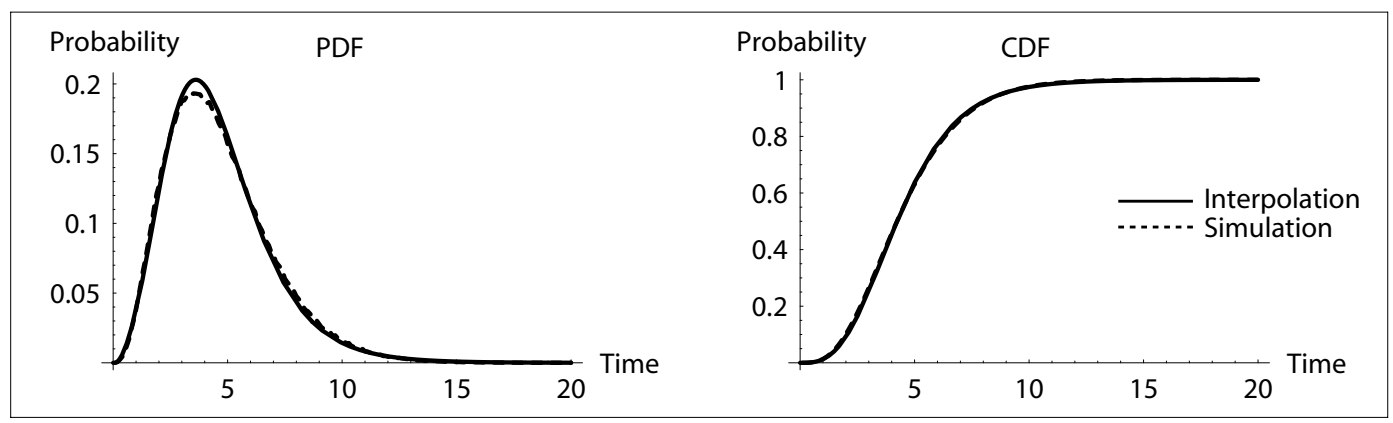

Figure 8: $\mathrm{CDF}$ and PDF of the serial line $\left(\rho=0.5, C^{2}=0.75,0.75,0.75,0.75\right)$. 


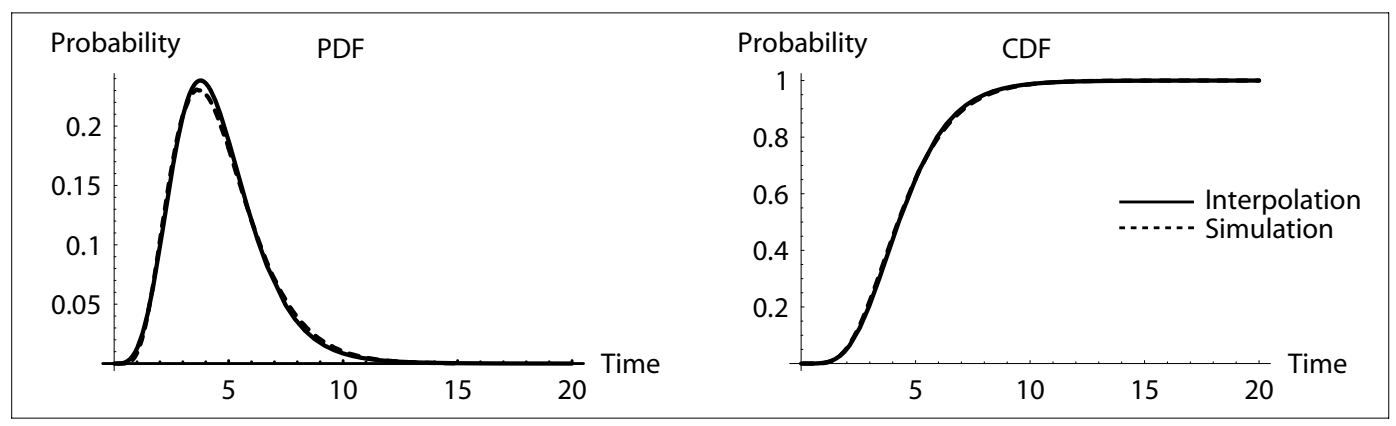

Figure 9: $\mathrm{CDF}$ and PDF of the serial line $\left(\rho=0.5, C^{2}=0.4,0.6,0.26,0.8\right)$.

Table 5: Anderson-Darling tests for the serial line test case.

\begin{tabular}{lcccccccccc}
\hline & & & & & & & & \multicolumn{2}{c}{$\%$ difference } \\
\cline { 7 - 9 } & & $C_{s}^{2}(1)$ & $C_{s}^{2}(2)$ & $C_{s}^{2}(3)$ & $\mathrm{A}-\mathrm{D}$ & $\alpha=5 \%$ & Decision & $90^{\text {th }}$ & $95^{\text {th }}$ \\
\hline \multirow{2}{*}{0.85} & 0.75 & 0.75 & 0.75 & 0.75 & 1.691 & \multirow{2}{*}{0.492} & accept & 0.44 & -0.32 \\
& 0.4 & 0.6 & 0.26 & 0.8 & 1.529 & & accept & -1.15 & -0.38 \\
\hline \multirow{2}{*}{0.5} & 0.75 & 0.75 & 0.75 & 0.75 & 0.639 & & accept & 1.92 & -0.26 \\
& 0.4 & 0.6 & 0.26 & 0.8 & 1.542 & & accept & -1.65 & -1.88 \\
\hline
\end{tabular}

Example 4 To test the model when squared coefficients of variation are greater than one, consider the problem data in Table 6 . and the mean sojourn time from the intuitive model and the simulation for a sample 3-workstation serial line. As before, the model seems to produce good results.

Example 5 Consider the general acyclic queueing network with 4-workstations in Figure 5. Workstations 1 and 4 have 6 servers, and workstations 2 and 3 have 4 and 2 servers respectively. All servers have the same capacity $(E[T]=1.5)$, and interarrival times are adjusted to produce the appropriate utilization $(\rho=0.5,0.85)$.

Table 7 shows the mean sojourn time of the interpolation model and the simulation model in a 4-workstation general queueing network. Similar to the serial line case, the interpolation model gives better results under low utilization than under high utilization. The percent differences are all less than 5 percent. 
Table 6: A comparison of mean sojourn times for the intuitive model and simulation for a 3workstation serial line when $C^{2}>1$.

$$
E[T] \quad C^{2} \quad \rho \frac{\text { Mean sojourn time (hour) }}{\text { Simulation Intuitive }} \% \text { difference }
$$

\begin{tabular}{|c|c|c|c|c|c|c|}
\hline Interarrival & 0.5 & 1.2 & & \multirow{4}{*}{4.70} & \multirow{4}{*}{4.76} & \multirow{4}{*}{1.29} \\
\hline Workstation 1 & 1.5 & 1.5 & 0.5 & & & \\
\hline Workstation 2 & 1.5 & 1.6 & 0.5 & & & \\
\hline Workstation 3 & 1.5 & 1.4 & 0.5 & & & \\
\hline
\end{tabular}

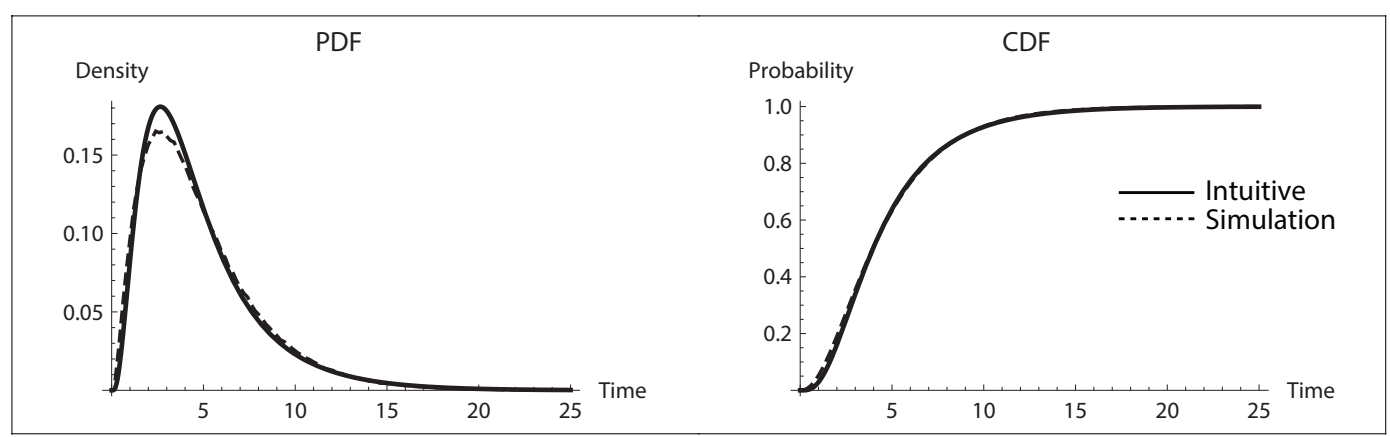

Figure 10: Sojourn time distributions for Example 4.

Figures 11-14 show distributions from the model and simulation. The Anderson-Darling tests are all acceptable and values for the $90^{t h}$ and $95^{t h}$ percentiles show fairly nice agreement.

Example 6 Consider a serial line with 3-workstations and 95 workers in the system. We assume that both the interarrival and service times follow general distributions. Table 9 shows the system information in detail.

We compare the mean sojourn time and distribution between the interpolation and simulation model. We also record computation time using a $2.4 \mathrm{GHz}$ Intel Core 2 Duo processer, and it takes 1.55 hours. Table 10 and Figure 15 show mean sojourn time and sojourn time distribution.

We see that the interpolation model also works well for the large scale system, but the computation time has increased significantly. As mentioned before, the size of the infinitesimal generator $K$ and the initial probability vector $\boldsymbol{\zeta}$, which decide the sojourn time distribution, are determined by 
Table 7: Mean sojourn times for a 4-workstation general network.

\begin{tabular}{ccccccccc}
\hline$\rho$ & $C_{a}^{2}$ & $C_{s}^{2}(1)$ & $C_{s}^{2}(2)$ & $C_{s}^{2}(3)$ & $C_{s}^{2}(4)$ & Interpolation & Simulation & $\%$ difference \\
\hline \multirow{4}{*}{0.85} & 1 & 1 & 1 & 1 & 1 & 8.99 & 8.91 & 0.89 \\
\cline { 2 - 9 } & 0.75 & 0.75 & 0.75 & 0.75 & 0.75 & 7.78 & 7.98 & -2.61 \\
\cline { 2 - 10 } & 0.57 & 0.7 & 0.6 & 0.6 & 0.9 & 7.31 & 7.49 & -2.42 \\
\hline & 0.45 & 0.75 & 0.4 & 0.4 & 0.9 & 6.73 & 7.05 & -4.48 \\
\hline & 0.4 & 0.6 & 0.26 & 0.26 & 0.8 & 6.38 & 6.68 & -4.46 \\
\hline & 0.33 & 0.33 & 0.33 & 0.33 & 0.33 & 6.11 & 6.27 & -2.62 \\
\hline \multirow{3}{*}{0.5} & 1 & 1 & 1 & 1 & 4.76 & 4.81 & -1.11 \\
\hline & 0.75 & 0.75 & 0.75 & 0.75 & 0.75 & 4.73 & 4.74 & -0.17 \\
\hline & 0.57 & 0.7 & 0.6 & 0.6 & 0.9 & 4.68 & 4.69 & -0.36 \\
\hline & 0.45 & 0.75 & 0.4 & 0.4 & 0.9 & 4.60 & 4.66 & -1.12 \\
\hline & 0.4 & 0.6 & 0.26 & 0.26 & 0.8 & 4.59 & 4.64 & -0.94 \\
\hline & 0.33 & 0.33 & 0.33 & 0.33 & 0.33 & 4.58 & 4.62 & -0.84 \\
\hline
\end{tabular}

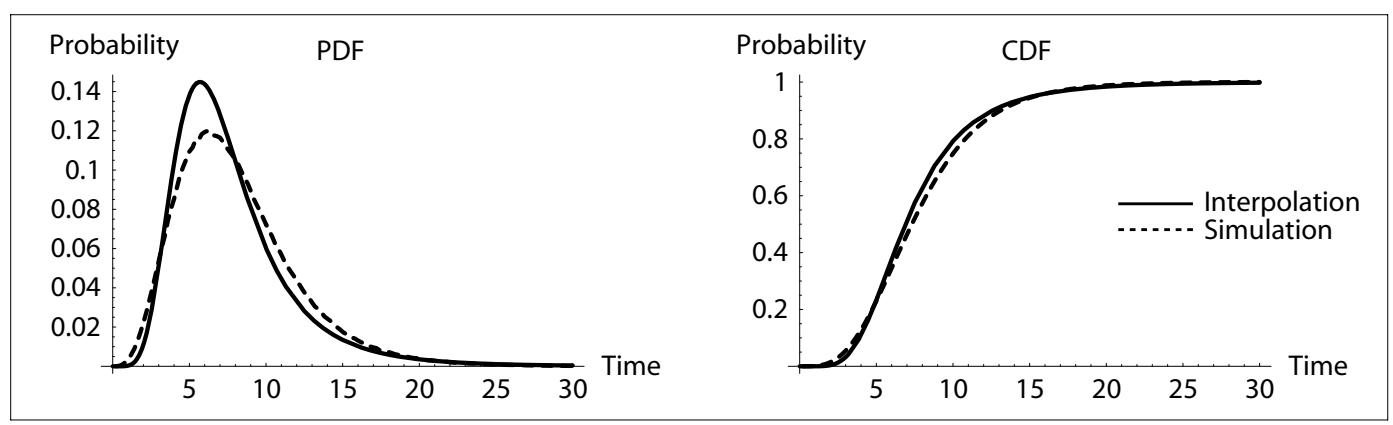

Figure 11: CDF and PDF of the general network $\left(\rho=0.85, C^{2}=0.75,0.75,0.75,0.75,0.75\right)$. 


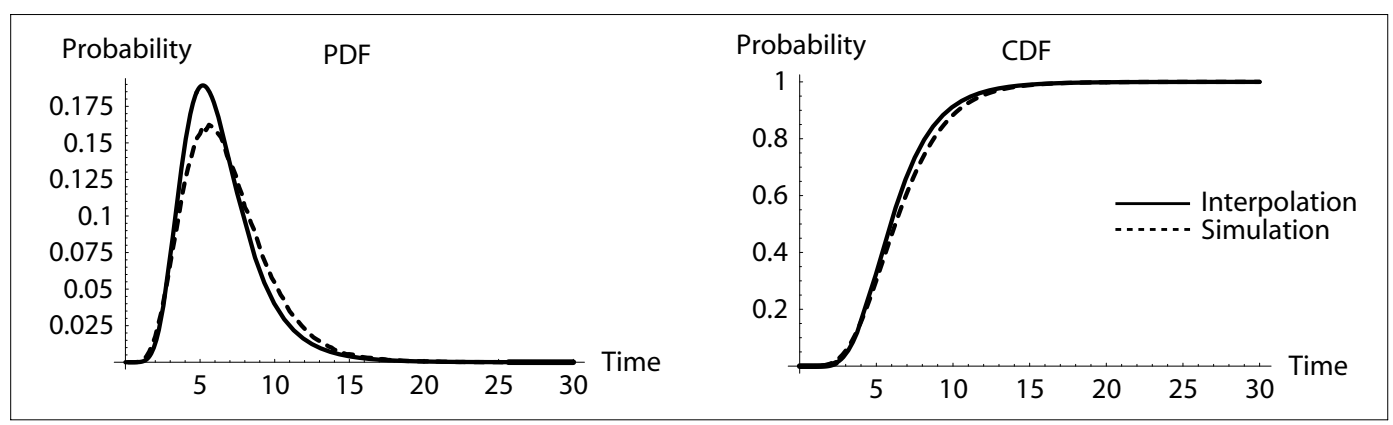

Figure 12: $\mathrm{CDF}$ and PDF of the general network $\left(\rho=0.85, C^{2}=0.4,0.6,0.26,0.26,0.8\right)$.

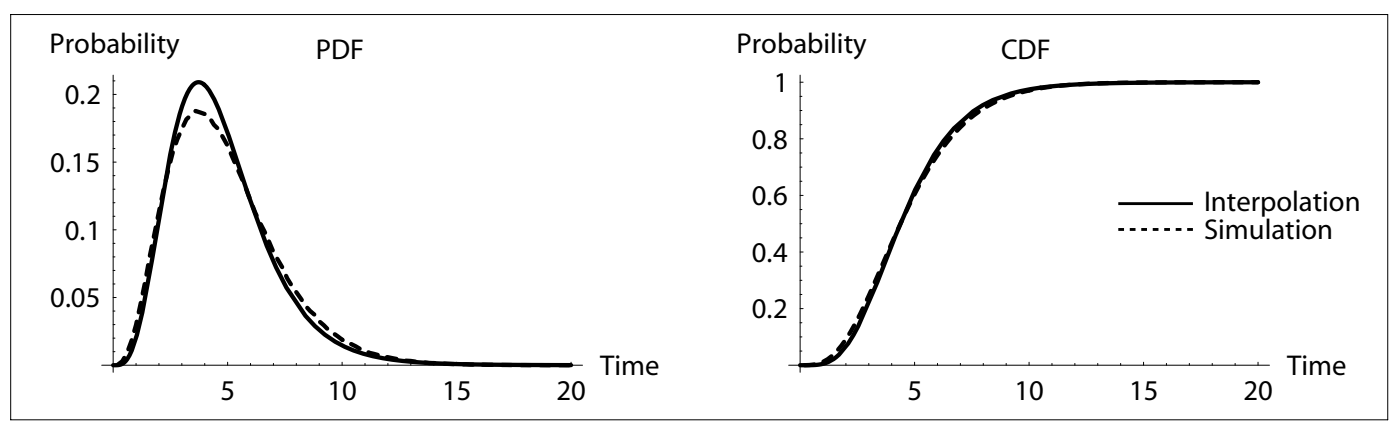

Figure 13: $\mathrm{CDF}$ and PDF of the general network $\left(\rho=0.5, C^{2}=0.75,0.75,0.75,0.75,0.75\right)$.

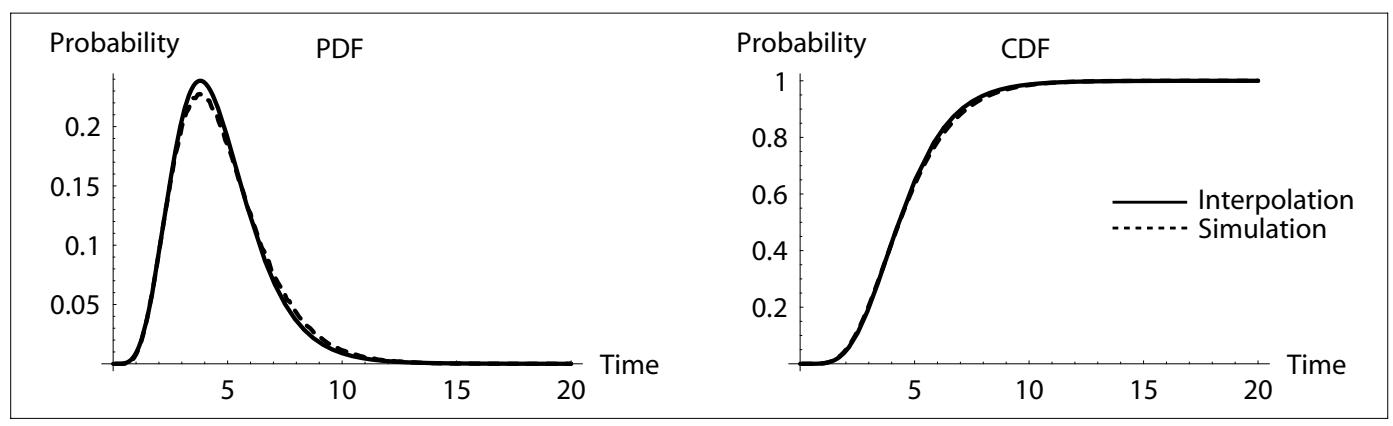

Figure 14: $\mathrm{CDF}$ and PDF of the general network $\left(\rho=0.5, C^{2}=0.4,0.6,0.26,0.26,0.8\right)$. 
Table 8: Anderson-Darling tests for the general queueing network.

\begin{tabular}{|c|c|c|c|c|c|c|c|c|c|c|}
\hline \multirow{2}{*}{$\rho$} & \multirow{2}{*}{$C_{a}^{2}$} & \multirow{2}{*}{$C_{s}^{2}(1)$} & \multirow{2}{*}{$C_{s}^{2}(2)$} & \multirow{2}{*}{$C_{s}^{2}(3)$} & \multirow{2}{*}{$C_{s}^{2}(4)$} & \multirow{2}{*}{ A-D } & \multirow{2}{*}{$\alpha=5 \%$} & \multirow{2}{*}{ Decision } & \multicolumn{2}{|c|}{$\%$ difference } \\
\hline & & & & & & & & & $90^{t h}$ & $95^{t h}$ \\
\hline \multirow{2}{*}{0.85} & 0.75 & 0.75 & 0.75 & 0.75 & 0.75 & 2.343 & \multirow{2}{*}{2.492} & accept & -4.02 & -1.33 \\
\hline & 0.4 & 0.6 & 0.26 & 0.26 & 0.8 & 2.046 & & accept & -5.70 & -5.27 \\
\hline \multirow{2}{*}{0.5} & 0.75 & 0.75 & 0.75 & 0.75 & 0.75 & 1.475 & \multirow{2}{*}{2.492} & accept & -3.57 & -3.28 \\
\hline & 0.4 & 0.6 & 0.26 & 0.26 & 0.8 & 1.454 & & accept & -2.93 & -3.05 \\
\hline
\end{tabular}

Table 9: The system information of the Example 6

\begin{tabular}{cccccc}
\hline & $E[T]$ & $C_{s}^{2}$ & $C_{a}^{2}$ & $\rho$ & Number of workers \\
\hline Interarrival & 0.23 & & 0.75 & & \\
Workstation 1 & 7.80 & 0.70 & & 0.85 & 40 \\
Workstation 2 & 4.50 & 0.70 & 0.78 & 25 \\
Workstation 3 & 5.80 & 0.80 & 0.84 & 30 \\
\hline
\end{tabular}

Table 10: Mean sojourn time for the Example 6.

\begin{tabular}{l} 
Interpolation \\
\hline
\end{tabular}

Figure 15: PDF and CDF of Example 6. 
the size of the matrix of the waiting time distribution $W$ and service time distribution $S$. Here, $W$ is determined by $\left(\begin{array}{c}m+c-1 \\ c\end{array}\right)$, where $m$ is the number of phases of the service time distribution and $c$ is the number of servers, and $S$ is determined only by $m$. An increase in $c$ affects mainly the size of the matrix $W$, and this creates a significant computational burden.

Example 7 Consider a serial line with 8-workstations and 91 workers in the system. We assume that both the interarrival and service times follow general distributions. Table 11 shows the system information in detail.

Table 11: The system information for Example 7.

\begin{tabular}{cccccc}
\hline & $E[T]$ & $C_{s}^{2}$ & $C_{a}^{2}$ & $\rho$ & Number of workers \\
\hline Interarrival & 0.23 & & 0.75 & & \\
Workstation 1 & 2.90 & 0.70 & & 0.84 & 15 \\
Workstation 2 & 2.50 & 0.60 & 0.91 & 12 \\
Workstation 3 & 1.40 & 0.80 & 0.87 & 7 \\
Workstation 4 & 1.50 & 0.65 & 0.82 & 8 \\
Workstation 5 & 2.75 & 0.50 & 0.91 & 13 \\
Workstation 6 & 2.20 & 0.85 & 0.87 & 11 \\
Workstation 7 & 3.10 & 0.70 & 0.79 & 17 \\
Workstation 8 & 1.50 & 0.60 & 0.82 & 8 \\
\hline
\end{tabular}

We compare the mean sojourn time and distribution between the interpolation and simulation model. Table 12 and Figure 16 show the mean sojourn time and sojourn time distribution, but the results are not as encouraging as before. The percent difference in the means is greater than $5 \%$, and the percent differences in the $90^{\text {th }}$ and $95^{\text {th }}$ percentiles are also greater than $5 \%$. However, computation time is a reasonable 13 minutes because the number of servers in each workstation is not too large, and the matrix size for each waiting time distribution is of reasonable size. Results for this example suggest the quality of the approximation decreases as the length of the serial line increases. 
Table 12: Mean sojourn time for Example 7.

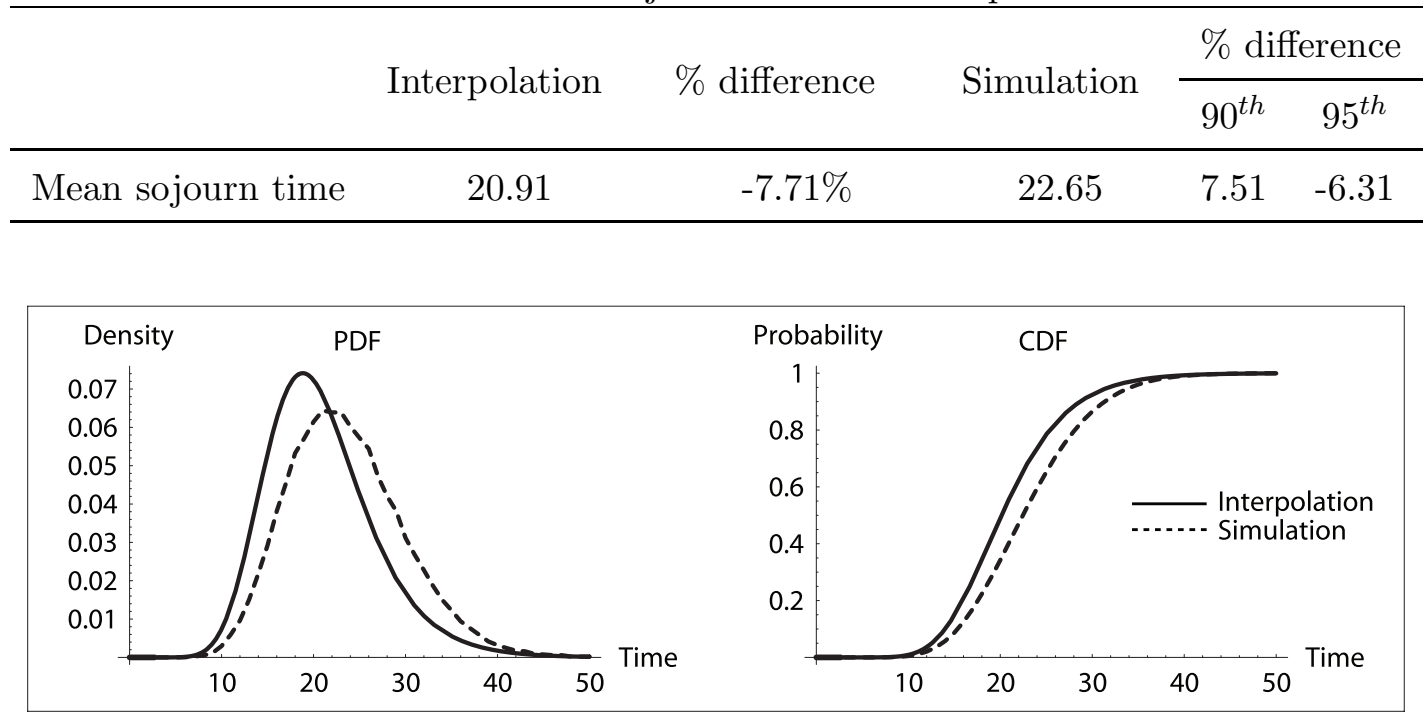

Figure 16: PDF and CDF for the long serial line in Example 7.

\section{Conclusions}

We have developed an approximation model for the steady state sojourn time distribution of entities arriving to an acyclic network of multi-server queues. In general, distributions produced by the model agree well with simulations of identical systems for the cases we have tested. Means of the approximated distributions were generally within 3 percent of the simulated means, and the distributions produced met the requirements of the Anderson-Darling test. The test of a long serial line ( 8 workstations) produced less satisfactory results, suggesting that the quality of the results would degrade as the size and complexity of the network increases. Nevertheless, the model could be used by any organization interested in making probabilistic statements about the time a job or customer will spend in a network of multi-server queues.

The method we have developed requires only basic statistics about interarrival and processing times, which can take on any general distribution. For processing times this seems straightforward, but an acknowledged weakness of the model - at least for many practical systems - is the assumption of stationary interarrival times. We suspect that for many systems, this assumption is appropriate and for some it is plainly inappropriate, but we have no basis to make conclusive statements. Users 
facing a practical problem with highly non-stationary interarrival times would have to verify the approximation model with simulation or with empirical data, in order to proceed with confidence.

Acknowledgements We thank Drs. Asmussen and Møller for computer code that facilitated the use of their models. This research was generously supported by the Office of Naval Research.

\section{References}

Asmussen, S. and Møller, J. R. (2001). Calculation of the Steady State Waiting Time Distribution in GI/PH/c and MAP/PH/c Queues. Queueing Systems, 37:9-29.

Asmussen, S. and O'Cinneide, C. A. (1998). Representations for matrix-geometric and matrixexponential steady-state distributions with applications to many-server queues. Stochastic Models, 14:369-387.

Curry, G. L. and Feldman, R. M. (2009). Manufacturing Systems Modeling and Analysis. SpringerVerlag.

Gue, K. R. and Kim, H. H. (2012). Predicting Departure Times in Multi-Stage Queueing Systems. Computers \& Operations Research, 39(7):1734-1744.

Law, A. M. and Kelton, W. D. (2000). Simulation Modeling and Analysis. McGraw-Hill, third edition.

Luh, H. and Zheng, Z. X. (2005). PH/PH/1 Queueing Models in Mathematica for Performance Evaluation. International Journal of Operations Research, 2(2):81-88.

Mandelbaum, A., Massey, W. A., Reiman, M. I., Stolyar, A., and Rider, B. (2002). Queue Lengths and Waiting Times for Multiserver Queues with Abandonment and Retrials. Telecommunication Systems, 21:149-171.

Neuts, M. F. (1981). Matrix-Geometric Solutions in Stochastic Models: An Algorithmic Approach. Johns Hopkins University Press, Baltimore, Maryland.

Neuts, M. F. (1989). Structured Stochastic Matrices of M/G/1 type and their Applications. Marcel Dekker, Inc., New York, New York. 
Rueda, J. E. (2003). The $P h_{t} / P h_{t} / \mathrm{s} / \mathrm{c}$ Queueing Model and Approximation. Master's thesis, Virginia Tech, Blacksburg, VA.

Sauer, C. and Chandy, K. (1975). Approximate analysis of central server models. IBM Journal of Research and Development, 19:301-313.

Sengupta, B. (1989). Markov processes whose steady state distribution is matrix-exponential with an application to the GI/PH/1 queue. Advances in Applied Probability, 21:159-180.

Shanthikumar, J. and Sumita, O. (1988). Approximations for the Time Spent in a Dynamic Job Shop with Applications to Due Date Assignment. International Journal of Production Research, 26:1329-1352.

Tijms, H. C. (1994). Stochastic models: An Algorithmic Approach. John Wiley \& Sons, Chichester.

Whitt, W. (1983). The Queuing Network Analyzer. Bell Systems Technical Journal, 62(9):27792815.

Whitt, W. (1999). Predicting Queueing Delays. Management Science, 45(6):870-888.

Yoon, B. S. (1994). An approximation method for sojourn time distributions in general queueing networks. Journal of the Korean Operations and Management Science, 19(3):93-109.

You, J. U., Chung, M. Y., and Sung, D. K. (2002). An Approximation Method for Sojourn Time Distributions in Telecommunication Networks. Computer Communications, 25:1283-1296. 